\title{
Customized Titanium Reconstruction of Orbital Fractures Using a Mirroring Technique for Virtual Reconstruction and 3D Model Printing
}

\author{
Blumer, Michael ; Pejicic, Rada ; Gander, Thomas ; Johner, Jean Pierre ; Held, Ulrike ; Wagner,
} Maximilian Eberhard

\begin{abstract}
PURPOSE Early operative reconstruction using titanium mesh is a technique often used for preventing sequelae after an orbital fracture. We sought to examine the utility of patient-specific molding of the mesh with a biomodel via virtual mirroring of the nonaffected side. METHODS We retrospectively assessed the clinical and radiological outcomes of orbital fracture reconstruction using a customized titanium mesh shaped on 3D-printed biomodels in 34 unilateral orbital fracture cases. Preoperative virtual orbital reconstruction images, using the mirroring technique, were superimposed on postoperative 3D images, and clinical data from patient charts were analyzed. Orbital reconstructions were rated, and the intention to revise results intraoperatively, or during inpatient or outpatient phases, was assessed by 2 consultants and 2 residents. RESULTS We found that most fractures arose from falls of $<3$ meters or from interpersonal violence. Ophthalmic injuries included subconjunctival bleeding, ocular contusion, enophthalmos, and diplopia. Long-term sequelae at last followup were diplopia (8.8\%) and mild enophthalmos (11.8\%). CONCLUSION Interrater reliabilities regarding consultants' intention to revise results were substantial to almost perfect at any time point. Therefore, using the mirroring technique for the virtual reconstruction of a fractured orbit and a 3D-printed biomodel to customize commercial titanium implants yields good and reliable results, enhances surgical precision, and decreases the need for intraoperative revision, as well as long-term sequelae of orbital fractures.
\end{abstract}

DOI: https://doi.org/10.1016/j.joms.2020.09.001

Posted at the Zurich Open Repository and Archive, University of Zurich

ZORA URL: https://doi.org/10.5167/uzh-196241

Journal Article

Accepted Version

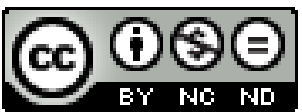

The following work is licensed under a Creative Commons: Attribution-NonCommercial-NoDerivatives 4.0 International (CC BY-NC-ND 4.0) License.

Originally published at:

Blumer, Michael; Pejicic, Rada; Gander, Thomas; Johner, Jean Pierre; Held, Ulrike; Wagner, Maximilian Eberhard (2021). Customized Titanium Reconstruction of Orbital Fractures Using a Mirroring Technique for Virtual Reconstruction and 3D Model Printing. Journal of Oral and Maxillofacial Surgery, 79(1):200.e1200.e9.

DOI: https://doi.org/10.1016/j.joms.2020.09.001 


\section{Customized Titanium Reconstruction of Orbital Fractures using a Mirroring Technique for Virtual Reconstruction and 3D Model Printing}

Dr. med. Dr. med. dent. Michael Blumer"1), M Dent Med Rada Pejicic ${ }^{2}$, PD Dr. med. Dr. med. dent. Thomas Gander ${ }^{1}$, med. pract. M Dent Med Jean Pierre Johner ${ }^{3)}$, PD Dr. rer. nat. Ulrike Held ${ }^{4)}$, Maximilian Eberhard Wagner, Dr. med. univ. Dr. med. dent. ${ }^{1)}$

1) Consultant, Clinic for Oral and Maxillofacial Surgery, University Hospital Zurich, Zurich, Switzerland

2) Consultant, Policlinic for Oral Surgery, Centre of Dental Medicine Zurich, Zurich, Switzerland

3) Resident, Clinic for Oral and Maxillofacial Surgery, University Hospital Zurich, Zurich, Switzerland

4) Senior Researcher, Department of Biostatistics and Epidemiology, Biostatistics and Prevention Institute, University of Zurich, Zurich, Switzerland

\section{Corresponding author:}

Michael Blumer

Dr. med. Dr. med. dent.

UniversitätsSpital Zürich

Klinik für Mund-, Kiefer- und Gesichtschirurgie

Frauenklinikstrasse 24

8091 Zürich

+41432539575

Michael.Blumer@usz.ch 


\section{Abstract \\ Purpose}

Early operative reconstruction using titanium mesh is a technique often used for preventing sequelae after orbital fracture. We sought to examine the utility of patient-specific molding of the mesh with a biomodel via virtual mirroring of the non-affected side.

\section{Methods}

We retrospectively assessed the clinical and radiological outcomes of orbital fracture reconstruction using a customized titanium mesh shaped on 3D-printed biomodels in 34 unilateral orbital fracture cases. Preoperative virtual orbital reconstruction images, using the mirroring technique, were superimposed on postoperative 3D images, and clinical data from patient charts were analyzed. Orbital reconstructions were rated and the intention to revise results intraoperatively, or during inpatient or outpatient phases, was assessed by 2 consultants and 2 residents.

\section{Results}

We found that most fractures arose from falls of $<3$ meters or from interpersonal violence. Ophthalmic injuries included subconjunctival bleeding, ocular contusion, enophthalmos, and diplopia. Long-term sequelae at last follow-up were diplopia (8.8\%) and mild enophthalmos $(11.8 \%)$.

\section{Conclusion}

Interrater reliabilities regarding consultants' intention to revise results were substantial to almost perfect at any timepoint. Therefore, using the mirroring technique for virtual reconstruction of a fractured orbit and a 3D-printed biomodel to customize commercial titanium implants yields good and reliable results, enhances surgical precision, and decreases the need for intraoperative revision as well as long-term sequelae of orbital fracture. 


\section{Introduction}

Fractures of the orbit alone and in combination with other fractures are common in cases of midface trauma. The orbital floor is affected in up to $57 \%$ and the medial wall in $12 \%$ of midface fractures [1]. Early operative reconstruction is recommended to prevent sequelae, such as enophthalmos or diplopia, which are present in up to $20 \%$ of cases preoperatively [1,2].

Although a recent study found no correlation between preoperative orbital volume change and late diplopia and enophthalmos in surgically treated patients [3], anatomical repair is preferable in patients who need operative treatment $[4,5]$.

For small defects, polydioxanone (PDS) sheets are commonly used, while for larger defects, commercial (preformed and standard) titanium meshes may be used as rigid support $[5,6]$. Recently, patient-specific reconstruction using a technique that involves mirroring of the nonaffected side and virtual reconstruction has become more popular [7, 8]. Customizing commercial titanium meshes using 3D-printed anatomical models is 1 option [9-12]. Another fully automated workflow involves virtual design of a patient-specific implant (PSI) and additive manufacturing of the implant from titanium [13-16]. Given the high costs of PSIs [5, 6], we consider that they should be primarily used for complex orbital fractures.

Consequently, 3D-printed models for preoperative and intraoperative customizing the titanium mesh is an interesting alternative. Intraoperative quality control may be achieved through infrared-based navigation or 3D intraoperative imaging. Both techniques enhance the quality of the reconstruction $[7,14,17-21]$.

The present retrospective study analyzed the clinical and radiological results of using commercial titanium meshes that were planned and customized on 3D-printed biomodels preor intraoperatively. Furthermore, the intention to revise the surgical result intraoperatively by means of 3D imaging was analyzed by 4 different examiners. 


\section{Methods}

The clinical records of the UniversitätsSpital Zürich was searched for patients who underwent orbital reconstruction due to a unilateral orbital fracture, between October 2014 and June 2016. Inclusion criteria were defined as unilateral orbital fracture, and orbital reconstruction with a titanium mesh that was customized on a 3D-printed biomodel using a preoperative virtual reconstruction workflow. Informed consent was obtained from all participating patients, as was ethical approval from the responsible ethics committee at KEK Zürich (approval: 201800953). The study adhered to the tenets of the Declaration of Helsinki regarding ethical principles for medical research involving human subjects.

Of the 39 patients, 5 patients had to be excluded, as informed consent was lacking, leaving 34 patients for analysis of their clinical and radiological data. Clinical examination was performed by staff members of the Oral and Maxillofacial Department preoperatively (on the day of admission to the hospital), postoperatively at the inpatient stage (usually the day of dismissal), and in the outpatient stage at the last patient visit (end of follow-up checks). All patients also underwent pre- and postoperative ophthalmological examinations (before surgery and before hospital dismissal) performed by a staff member of the Ophthalmological Department.

\section{Surgical procedure}

The transconjunctival retro-septal approach was used to dissect the orbital fractures in 32 patients. 1 patient had a soft tissue lesion of the lid, which was used as approach, and in the other case, a transconjunctival preseptal approach was used.

Virtual reconstruction using the mirroring technique was applied preoperatively in the iPlan ENT software (Brainlab, Feldkirchen, Germany), and 3D models were printed using an industrial style printer (Objet 260V, Stratasys Ltd., Eden Prairie, MN, USA). The 3D-printed biomodel was sterilized in the operation theatre for customizing or for corrections. The orbital implant (MatrixMIDFACETM, Synthes GmbH, Oberdorf, Switzerland) was either customized 
by molding to the biomodel prior to the sterilization process or a sterile stock implant was customized on the sterilized biomodels, intraoperatively (Fig. 1).

Postoperatively, all patients underwent 3D imaging (either conventional or cone-beam CT). Visual acuity and pupillary reaction were observed every hour for the first 6 hours, then every 2 hours for the next 24 hours post-surgery, in order to detect raised intraorbital pressure due to swelling or bleeding. Clinical follow-up was performed from 1 month to 30 months, depending on symptoms.

\section{Postoperative measurements}

Postoperative computed tomography (CT) images were merged in the iPlan software and the superimposition of reconstructed orbits with the virtual plan was analyzed and rated independently by 4 examiners (2 experienced consultants and 2 resident doctors) (Figs. 2 \& 3). Fleiss's $\mathrm{k}$ was calculated to quantify the agreement between raters. The intention of a surgeon to revise the surgical results was analyzed on the postoperative superimposed images for the following timepoints: intraoperative, in-hospital stay, or outpatient follow-up.

These intentions for revising the radiological results were compared to those in a previous study [7] with a similar experimental setup, in which non-preformed titanium, preformed titanium, and PDS materials were used for orbital reconstruction. This was done to analyze the effectiveness of customizing titanium implants using biomodels.

\section{Statistical analysis}

Descriptive statistics included mean and standard deviation for continuous variables, as well as number and percentage of total for the categorical variables. Fleiss' $\mathrm{k}$ values were reported with $95 \%$ confidence intervals $(\mathrm{Cl})$. For the comparison of continuous variables between independent groups, the Wilcoxon test was used. Chi-squared tests were used for the comparison of categorical variables across groups. Statistical analyses were performed using Excel software (Microsoft Corporation, Redmond, USA) and the R software (R Foundation, 
Wien, Austria) [22]. The results of the study are considered exploratory and the evidence was quantified on continuous scale.

\section{Results}

Most included patients were male and the mean age was 45 years. The left orbit was affected more frequently and the mean time from trauma to operative reconstruction was 7 days (Table 1). There were $10(29.4 \%)$ cases with a pure orbital fracture, whereas 24 (70.6\%) cases had associated midface fractures. Table 2 shows the overall classification of fractures.

Most orbital fractures originated from a fall of under 3 meters (13 cases, $38.2 \%$ ), followed by violence (10 cases, 29.4\%). 3 of the patients who fell from a height of more than 3 meters sustained at least a multiwall fracture of the orbit. The details of the trauma are shown in Table 3.

Only 9 cases $(26.5 \%)$ had no ophthalmic injuries. The associated ophthalmic injuries are shown in Table 4.

When we analyzed binocular diplopia in a preoperative and postoperative inpatient as well as a postoperative outpatient setting, we identified 2 patients $(5.9 \%)$ who had no diplopia preoperatively, but who had persistent diplopia at the last follow-up visit. In 1 case (2.9\%), preoperative diplopia persisted postoperatively. In 6 cases (17.6\%), preoperative diplopia resolved postoperatively, and in 11 cases (32.4\%), preoperative diplopia resolved by the last follow-up visit (Table 5). In total, 11 patients (32.04\%) showed binocular diplopia in the inpatient and only $3(8.8 \%)$ in the outpatient setting.

Patients with persistent binocular diplopia had 1 multiwall orbital fracture, while the other 2 patients had multiple complex midface fractures in addition to orbital fractures. One of these 2 cases had to be revised for orbital reconstruction; this was the only case who underwent revision surgery. 
Other long-term sequelae, such as enophthalmos or exophthalmos, were rare; only 4 cases with enophthalmos (11.8\%) were detected. In these cases, enophthalmos was mild, with a 1mm difference according to Naugle ophthalmometry.

The group with pure orbital fractures differed from that with a combination of orbital and other midface fractures only in terms of operation time $(p=0.003)$. The mean operation time for reconstruction of pure orbital fractures was 102 minutes (median: 99 minutes). The group with pure orbital fractures had more preoperative enophthalmos $(4 ; 40 \%)$ and postoperative enophthalmos $(2 ; 20 \%)$ than the group with combined orbital and other midface fractures (preoperative: $3 ; 12.5 \%, p=0.2$; and postoperative: $2 ; 8.3 \%, p=0.7$ ). Preoperatively, the group with pure orbital fractures appeared to have more binocular diplopia $(5 ; 50 \%$ vs combination fractures: $7 ; 29.2 \% ; p=0.4)$; however, the difference did not reach statistical significance. Additionally, postoperatively, during the inpatient phase, the pure orbital fracture group was more susceptible to diplopia ( $5 ; 50 \%$ vs combination fractures: $6 ; 25 \% ; p=0.3$ ). This difference resolved during the outpatient phase. At the last patient visit, 1 case in the pure orbital fracture group (10\%) and 2 cases in the combined fractures group had diplopia (8.3\%, $\mathrm{p}=0.9)$

The interrater reliability for intention to revise was assessed at 3 timepoints and the 4 raters were divided into experienced consultants and residents ( $n=2$ each). Substantial to almost perfect interrater reliability for consultants were found using Fleiss's $\mathrm{K}$ for the intention to revise an operative result at any timepoint. These results are shown in Table 6 and Figure 4. Fleiss's $K$ for residents was only poor to fair.

\section{Discussion}

Customized stock titanium meshes for orbital reconstruction hold promise for an anatomical shape with normal function. In this retrospective study, we assessed the clinical and radiological outcomes of cases of orbital fracture that were reconstructed using titanium meshes, customized by molding to a 3D-printed biomodel based on a virtual reconstruction 
achieved by mirroring of the uninjured side. We showed that using this approach for customizing titanium mesh implants yielded good and reliable results, enhancing surgical precision and decreasing the need for intraoperative revision, as well as minimizing the longterm sequelae of orbital fracture.

In our study, orbital fractures were most often due to falls from heights under 3 meters and from interpersonal violence, and more males were affected; these findings were in line with those of other studies on midface fractures $[23,24]$. On the other hand, we had a sizeable proportion of cases due to falls from heights exceeding 3 meters ( $8.8 \% ; 3$ cases). This might be due to a bias caused by the inclusion criterion for this study, which focused on cases requiring customized titanium meshes for orbital reconstruction, which are more often used in complex or markedly large orbital defects. These 3 cases had multiwall defects with or without associated further midface fractures.

As result of trauma, binocular diplopia was present in 12 cases (35.3\%), enophthalmos in 7, and exophthalmos in 1 case in our study. The prevalence of diplopia was higher than that in a previous study of ophthalmic injuries accompanying midface fractures $(18.4 \%$ of these cases presented preoperative diplopia) [1], while enophthalmos and exophthalmos had a similar prevalence as in the abovementioned study. The higher prevalence of diplopia was probably due to our inclusion criteria, as the apparently larger orbital fractures in this study would be likely to lead to subsequent functional impairment. Interestingly, in $26.5 \%$ of cases, no associated ophthalmic injury was found. Nevertheless, we recommend an examination by an ophthalmologist for all orbital fracture cases, not least for forensic reasons.

In our study, 6 cases of diplopia were resolved after surgery, whereas it remained in another 6 and new binocular diplopia emerged in 5 cases postoperatively; consequently, 11 patients (32.4\%) presented diplopia postoperatively. In most cases, this functional impairment is due to perioperative swelling, and vanishes once the swelling has resolved [25]. Indeed, at the last patient visit, diplopia had disappeared in 11 of the initial 12 patients with diplopia. In 1 patient, preoperative binocular diplopia persisted and in 2 patients, diplopia developed postoperatively (3 cases; 8.8\%). A recent study analyzing postoperative diplopia in orbital and orbitomalar 
fractures showed a preoperative prevalence of diplopia of $38.4 \%$ and postoperative diplopia prevalence of $20.9 \%$ [26]. Furthermore, this study showed a correlation between the presence of preoperative and postoperative diplopia. Moreover, in that study, 4 patients with no preoperative diplopia developed diplopia postoperatively $(2.6 \%)$. The rate was higher in our study (5.8\%, 2 cases); 1 of these cases was the sole patient in our study requiring revision for complex midface and orbital fractures. Multiple surgeries are also considered to make postoperative diplopia more likely.

Binocular diplopia had a low prevalence in our population sample (8.8\%; 3 cases). Previous reports have indicated that the postoperative prevalence of diplopia ranges between $8 \%$ and $42 \%[26,27]$. Thus, the method of customizing orbital implants by molding to 3D-printed biomodels of the mirrored healthy orbit presents few postoperative diplopia complications. Furthermore the diplopia was not in the primary gaze in these patients, but only in the extreme view.

Four patients had long-term enophthalmos (11.8\%), which is on the lower end of the range reported in the literature (7-27\%) [27]. The literature presents evidence that extension of the fracture to the medial wall or the conjunction of the orbital floor and medial wall increases the likelihood of enophthalmos; through correct anatomical reconstruction and adequate surgery, no worse results are expected [27, 28]. The enophthalmos detected in our study was measured by Naugle ophthalmometry, which has been shown to be equivalent to the Hertel method [29]. The difference between healthy and operated sides in our cases was only $1 \mathrm{~mm}$, whereas enophthalmos becomes clinically recognizable when the difference reaches 3-4 mm; patients with a difference of $2 \mathrm{~mm}$ and less have a normal appearance [30].

There was no evidence of a statistically significant difference between the groups with pure orbital fractures and those with a combination of orbital and other midface fractures. The group with pure orbital fractures seemed to be more prone to enophthalmos and binocular diplopia in the postoperative inpatient setting, but without statistical significance. Diplopia differences between the groups resolved in the outpatient setting (pure orbital group: $10 \%$ and combination fracture group: $8.3 \%$ ). Another study found differences between such groups, 
showing a higher prevalence of diplopia in the pure orbital fracture group [26]. Our study had a much longer follow-up than the previous study, which might explain this finding.

Our patients were operated on average within 7 days post-trauma (SD: 4.1 days). The literature agrees that more favorable results are obtained with early surgery. A recent metaanalysis showed that early surgery is significantly associated with resolution of vertical dystopia, postoperative enophthalmos, and motility restriction [31].

The 4 raters stated that they would have revised the intraoperative results based on 3D patient images and virtual reconstruction images in $20.6-50 \%$ of cases. This intention for revision decreased to $2.9-11.8 \%$ in the inpatient setting, and to $0-2.9 \%$ in the outpatient setting. In a similar study in 2015, 4 experienced raters were asked about their intention to revise results, obtained with PDS sheets and stock titanium implants, based on 3D patient imaging and virtual reconstruction imaging, and reported an intention to revise of $41.2-58.8 \%$ [7]. The intention to revise in the inpatient setting was $13.7-41.2 \%$ in the 2015 study, whereas it was decreased through customization using a 3D-printed biomodel to $2.9-11.8 \%$ in the present study. No difference was found between the studies in the outpatient setting (2015: 0-3.9\% revision; present study: $0-2.9 \%$ revision). Thus, by customizing titanium orbital implants on a 3Dprinted biomodel, the surgical precision was increased and the intention to revise followwas decreased. A recent study analyzed the effects of intraoperative CT and rapid prototyping on orbital fracture surgery and found an intraoperative revision rate of $22.7 \%$ and postoperative revision rate of $3.3 \%$ [32]. These values correspond well with the intention to revise of the 2 experienced consultants in our study.

Nevertheless, using Fleiss's $\mathrm{k}$ to assess interrater reliability, only fair to moderate interrater reliability was found [33]. Dividing the raters into consultants and residents, we found substantial to almost perfect interrater reliability for the consultants, but poor to fair reliability for the residents. Thus, even though intraoperative 3D imaging and the aid of virtual reconstruction is useful, a thorough understanding of the surgical procedure is required, and surgical experience is requisite for obtaining superior results in orbital surgery, as confirmed in another study [34]. 
When rating the actual orbital reconstruction, as compared to the planned virtual reconstruction, the consultants rated $79.4 \%$ as good reconstructions, with substantial interrater reliability. Interestingly, the raters stated that they would revise 4 cases, even though they rated the reconstructions as being in line with the preoperative planning. Therefore, intraoperative 3D imaging and instant superimposition with virtual reconstructions seemed to increase the surgeons' expectations of themselves. Further studies will be needed to determine whether this leads to overcorrection.

This study was limited in terms of its retrospective design and small sample size; however, these factors also facilitated the feasibility of conducting analyses on using 3D software images and interrater reliability.

In conclusion, our results showed that it is feasible to achieve good and reliable results by using the mirroring technique for virtual reconstruction of a fractured orbit and for 3D-printing a biomodel for customizing stock titanium orbital implants. This approach seems to enhance surgical precision and decrease the intention to revise results intraoperatively, as compared to a previous study. Long-term sequelae, such as enophthalmos and persistent diplopia were low in this study, and when present, was of little clinical significance. Therefore, intraoperative 3D imaging and superimposition of patient images with a preoperative virtual reconstruction image may further improve this delicate surgical field. Instant quality control and surgical revision is thereby made possible. Moreover, this approach may also contribute to education of oral and maxillofacial surgery residents. 


\section{References}

1. Blumer M, Rostetter C, Johner J-P, Ebner JJ, Wiedemeier D, Rücker M, Gander T: Associated Ophthalmic Injuries in Patients With Fractures of the Midface. Craniomaxillofacial Trauma \& Reconstruction 0:1943387520922056, 2020

2. Bartoli D, Fadda MT, Battisti A, Cassoni A, Pagnoni M, Riccardi E, Sanzi M, Valentini V: Retrospective analysis of 301 patients with orbital floor fracture. J Craniomaxillofac Surg 43:244, 2015

3. Schönegg D, Wagner M, Schumann P, Essig H, Seifert B, Rücker M, Gander TJJoC-MS: Correlation between increased orbital volume and enophthalmos and diplopia in patients with fractures of the orbital floor or the medial orbital wall. 46:1544, 2018

4. Kim JS, Lee BW, Scawn RL, Korn BS, Kikkawa DO: Secondary Orbital Reconstruction in Patients with Prior Orbital Fracture Repair. Ophthalmic Plast Reconstr Surg 32:447, 2016

5. Nkenke E, Vairaktaris E, Spitzer M, Kramer M, Stamminger M, Holbach L, Knipfer C, Stelzle F: Secondary reconstruction of posttraumatic enophthalmos: prefabricated implants vs titanium mesh. Arch Facial Plast Surg 13:271, 2011

6. Baumann A, Sinko K, Dorner G: Late Reconstruction of the Orbit With PatientSpecific Implants Using Computer-Aided Planning and Navigation. J Oral Maxillofac Surg 73:S101, 2015

7. Blumer M, Gander T, Kruse Gujer A, Seifert B, Rucker M, Lubbers HT: Influence of Mirrored Computed Tomograms on Decision-Making for Revising Surgically Treated Orbital Floor Fractures. J Oral Maxillofac Surg 73:1982.e1, 2015

8. Gellrich NC, Schramm A, Hammer B, Rojas S, Cufi D, Lagreze W, Schmelzeisen R: Computer-assisted secondary reconstruction of unilateral posttraumatic orbital deformity. Plast Reconstr Surg 110:1417, 2002

9. Baino F: Biomaterials and implants for orbital floor repair. Acta Biomaterialia $7: 3248,2011$ 
10. Kozakiewicz M, Elgalal M, Piotr L, Broniarczyk-Loba A, Stefanczyk L: Treatment with individual orbital wall implants in humans -1-Year ophthalmologic evaluation. Journal of Cranio-Maxillofacial Surgery 39:30, 2011

11. Zhang X, Chen W, Luo TY, Ma J, Dong Z, Cao G, Xu JK, Liu BY, Zhang QR, Zhang SL: Application of Three-Dimensional Printing Technology in the Orbital Blowout Fracture Reconstruction. J Craniofac Surg 30:1825, 2019

12. Zimmerer RM, Ellis E, Aniceto GS, Schramm A, Wagner MEH, Grant MP, Cornelius C-P, Strong EB, Rana M, Chye LT, Calle AR, Wilde F, Perez D, Tavassol F, Bittermann G, Mahoney NR, Alamillos MR, Bašić J, Dittmann J, Rasse M, Gellrich $\mathrm{N}-\mathrm{C}$ : A prospective multicenter study to compare the precision of posttraumatic internal orbital reconstruction with standard preformed and individualized orbital implants. Journal of Cranio-Maxillofacial Surgery 44:1485, 2016

13. Gander T, Essig H, Metzler P, Lindhorst D, Dubois L, Rücker M, Schumann PJJoC-MS: Patient specific implants (PSI) in reconstruction of orbital floor and wall fractures. 43:126, 2015

14. Rana M, Chui $\mathrm{CH}$, Wagner M, Zimmerer R, Rana M, Gellrich NC: Increasing the accuracy of orbital reconstruction with selective laser-melted patient-specific implants combined with intraoperative navigation. J Oral Maxillofac Surg 73:1113, 2015

15. Rana M, Holtmann H, Rana M, Kanatas AN, Singh DD, Sproll CK, Kübler NR, Ipaktchi R, Hufendiek K, Gellrich NC: Primary orbital reconstruction with selective laser melted core patient-specific implants: overview of 100 patients. British Journal of Oral and Maxillofacial Surgery 57:782, 2019

16. Ruiters S, Mombaerts I: Applications of three-dimensional printing in orbital diseases and disorders. Curr Opin Ophthalmol 30:372, 2019

17. Schramm A, Gellrich N-C, Schmelzeisen R: Navigational surgery of the facial skeleton. Springer Science \& Business Media, 2007

18. Schramm A, Wilde FJH: Die computergestützte Gesichtsschädelrekonstruktion. 59:800, 2011 
19. Wilde F, Lorenz K, Ebner A-K, Krauss O, Mascha F, Schramm A:

Intraoperative Imaging With a 3D C-Arm System After Zygomatico-Orbital Complex Fracture Reduction. Journal of Oral and Maxillofacial Surgery 71:894, 2013

20. Wilde F, Schramm A: Intraoperative Imaging in Orbital and Midface Reconstruction. Facial plast Surg 30:545, 2014

21. Wagner M, Gander T, Blumer M, Valdec S, Schumann P, Essig H, Rücker MJP: Die CAD/CAM-Revolution in der kraniofazialen Rekonstruktion. 2019

22. Team RC: R: A Language and Environment for statistical computing. $R$ Foundation for Statistical Computing, Vienna, Austria., 2019

23. Blumer M, Kumalic S, Gander T, Lanzer M, Rostetter C, Rucker M, Lubbers HT: Retrospective analysis of 471 surgically treated zygomaticomaxillary complex fractures. J Craniomaxillofac Surg 46:269, 2018

24. Boffano P, Roccia F, Zavattero E, Dediol E, Uglesic V, Kovacic Z, Vesnaver A, Konstantinovic VS, Petrovic M, Stephens J, Kanzaria A, Bhatti N, Holmes S, Pechalova PF, Bakardjiev AG, Malanchuk VA, Kopchak AV, Galteland P, Mjoen E, Skjelbred P, Koudougou C, Mouallem G, Corre P, Loes S, Lekven N, Laverick S, Gordon P, Tamme T, Akermann S, Karagozoglu KH, Kommers SC, Forouzanfar T: European Maxillofacial Trauma (EURMAT) project: a multicentre and prospective study. J Craniomaxillofac Surg 43:62, 2015

25. Chen C-T, Huang F, Chen Y-R: Management of posttraumatic enophthalmos. Chang Gung medical journal 29:251, 2006

26. Gavin Clavero MA, Simón Sanz MV, Til AM, Jariod Ferrer ÚM: Factors Influencing Postsurgical Diplopia in Orbital Floor Fractures and Prevalence of Other Complications in a Series of Cases. Journal of Oral and Maxillofacial Surgery 76:1725, 2018

27. Boyette JR, Pemberton JD, Bonilla-Velez J: Management of orbital fractures: challenges and solutions. Clinical ophthalmology (Auckland, N.Z.) 9:2127, 2015 
28. Ordon AJ, Kozakiewicz M, Wilczynski M, Loba P: The influence of concomitant medial wall fracture on the results of orbital floor reconstruction. J Craniomaxillofac Surg 46:573, 2018

29. Cole HP, 3rd, Couvillion JT, Fink AJ, Haik BG, Kastl PR: Exophthalmometry: a comparative study of the Naugle and Hertel instruments. Ophthalmic Plast Reconstr Surg 13:189, 1997

30. Koo L, Hatton MP, Rubin PA: When is enophthalmos "significant"? Ophthalmic Plast Reconstr Surg 22:274, 2006

31. Jazayeri HE, Khavanin N, Yu JW, Lopez J, Ganjawalla KP, Shamliyan T, Tannyhill RJ, 3rd, Dorafshar AH: Does Early Repair of Orbital Fractures Result in Superior Patient Outcomes? A Systematic Review and Meta-Analysis. J Oral Maxillofac Surg 78:568, 2020

32. Nguyen E, Lockyer J, Erasmus J, Lim C: Improved Outcomes of Orbital Reconstruction With Intraoperative Imaging and Rapid Prototyping. J Oral Maxillofac Surg 77:1211, 2019

33. Landis JRK, G.G.: The measurement of observer agreement for categorical data. Biometrics 33:159, 1977

34. Hsieh T-Y, Said M, Dedhia R, Timbang M, Steele T, Strong E: Assessment of the Learning Curve for Virtual Surgical Planning in Orbital Fractures. Craniomaxillofacial Trauma \& Reconstruction 194338752092394, 2020 
Table legends

Table 1: Patient characteristics.

\begin{tabular}{lcc}
\hline $\mathbf{N}$ & 34 & \\
\hline Male & 23 & $67.6 \%$ \\
Age (y) & 45.3 & $22.5(\mathrm{SD})$ \\
Right side & 13 & $38.20 \%$ \\
Time to operation (days) & 7.1 & $4.1(\mathrm{SD})$ \\
\hline
\end{tabular}


Table 2: Descriptive statistics of fractures, multiple fractures are possible.

\begin{tabular}{lcccccc}
\hline $\begin{array}{l}\text { Orbital } \\
\text { Floor }\end{array}$ & $\begin{array}{c}\text { Medial } \\
\text { Wall }\end{array}$ & NOE & ZMC & Nasal & LeFort I & LeFort II \\
\hline $\mathbf{3 3}$ & $\mathbf{1 1}$ & $\mathbf{4}$ & $\mathbf{1 9}$ & $\mathbf{1 0}$ & $\mathbf{2}$ & $\mathbf{1}$ \\
$97.1 \%$ & $32.4 \%$ & $11.8 \%$ & $55.9 \%$ & $29.4 \%$ & $5.9 \%$ & $2.9 \%$ \\
\hline
\end{tabular}

NOE, Naso-Orbital-Ethmoid Complex; ZMC, Zygomatico-Maxillary Complex 
Table 3: Etiology of orbital fractures.

Etiology N

Fall under $\mathbf{3} \mathbf{m}$ 13

$38.2 \%$

Interpersonal violence 10

$29.4 \%$

Sports 5

$14.7 \%$

Fall over $3 \mathrm{~m}$ 3

$8.8 \%$

Traffic accicent (car) 2

$5.9 \%$

Traffic accident (bicycle) 1 $2.9 \%$ 
Table 4: Preoperative ophthalmological findings.

$\begin{array}{ll}\text { Finding } & \mathrm{N} / \%\end{array}$

Subconjunctival bleeding

$26.4 \%$

Ocular contusion

Optic neuropathy

Bulbar perforation

$2.9 \%$

Binocular diplopia

Enophthalmos

$20.6 \%$

Exophthalmos

$2.9 \%$

No associated ophthalmic injury

9

$26.5 \%$ 
Table 5: Clinical course of binocular diplopia.

\section{Preoperative Postoperative}

\section{Inpatient Outpatient}

Overall

Improvement

Stable

Worsened
12

6

11

6

1

5

2 
Table 6: Estimated Fleiss $\mathrm{k}$ values (lower and upper 95\% confidence interval) for agreement between 4 raters ( 2 consultants and 2 residents)

\begin{tabular}{lcccccccccc}
\hline & \multicolumn{3}{c}{ All 4 Raters } & \multicolumn{3}{c}{ Consultants } & \multicolumn{3}{c}{ Residents } \\
& $\mathrm{K}$ & lower & upper & $\mathrm{K}$ & lower & upper & $\mathrm{K}$ & lower & upper \\
\hline Intraoperative & 0.36 & 0.22 & 0.5 & 0.67 & 0.34 & 1 & -0.03 & -0.37 & 0.31 \\
Inpatient & 0.55 & 0.41 & 0.69 & 1 & 0.66 & 1 & 0.35 & 0.02 & 0.69 \\
Outpatient & 0.2 & 0.07 & 0.34 & 1 & 0.66 & 1 & -0.01 & -0.35 & 0.32 \\
Reconstruction & 0.24 & 0.11 & 0.38 & 0.71 & 0.38 & 1 & -0.08 & -0.41 & 0.26 \\
quality & & & & & & & & & & \\
\hline
\end{tabular}




\section{Figure legends}

Fig. 1: Customized stock titanium orbital implant in a frontal view (Fig. 1A) and sagittal view (Fig. 1B). The double-S form of the natural orbital cone can be easily and reliably reconstructed. The 3D-printed, sterilized biomodel in the operation theatre (Fig. 1C); a different case showing a customized stock titanium orbital implant in the 3D-printed sterilized biomodels, intraoperatively (Fig. 1D).

Fig. 2: Example of virtual reconstruction of the left fractured orbit (red) derived from the right non-affected orbit (green) using the mirroring technique. The postoperative 3D image was subsequently superimposed on this preoperatively planned image and rated. This example was rated as good (in line with preoperative planning). Axial plane (Fig. 2A), sagittal plane (Fig. 2B), and coronal plane (Fig. 2C).

Fig. 3: Example of an orbital reconstruction rated as poor (not in line with preoperative planning). This example shows an enlarged orbit, not respecting anatomical landmarks, which was partially due to an incorrectly reduced zygomaticomaxillary complex. This case was revised; nevertheless, functional impairment persisted despite correct reduction and reconstruction. Axial plane (Fig. 3A), sagittal plane (Fig. 3B), and coronal plane (Fig. 3C).

Fig. 4: Absolute numbers for intention to revise the surgical result at 3 timepoints (Fig. 4A: intraoperative; Fig. 4B: inpatient; Fig. 4C: outpatient) and assessment of reconstruction quality by the 4 examiners (Fig. 4D). 


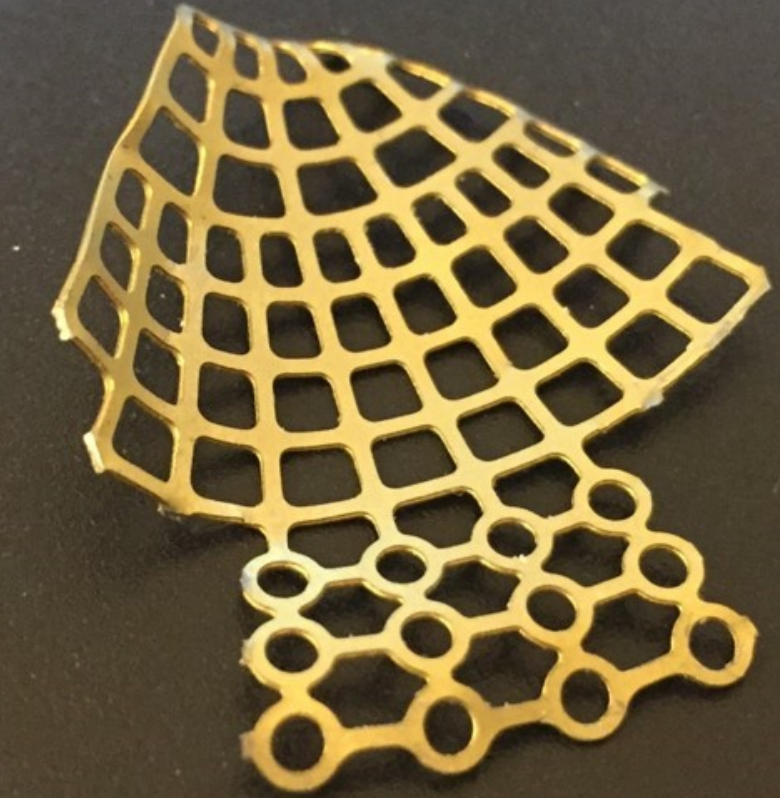




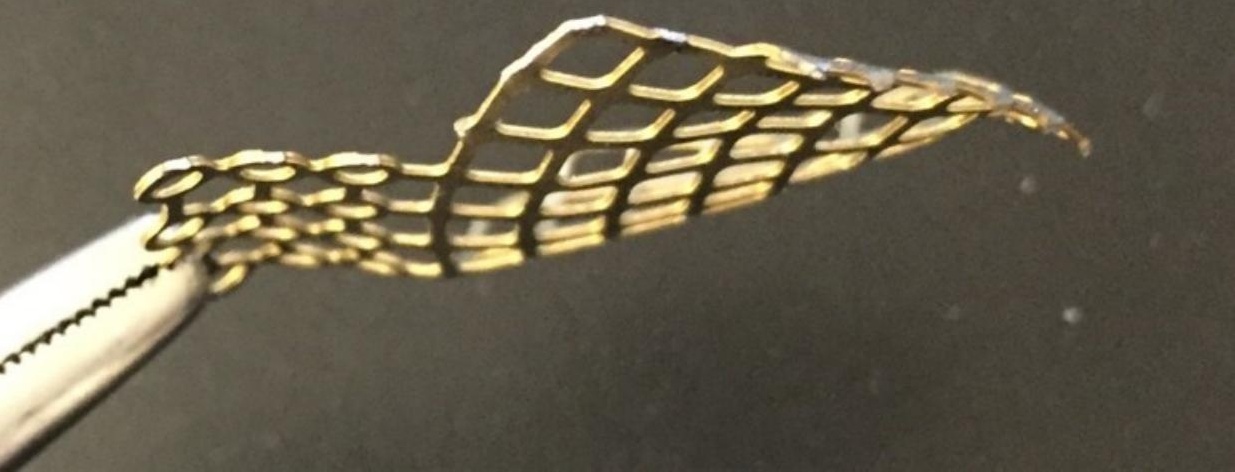




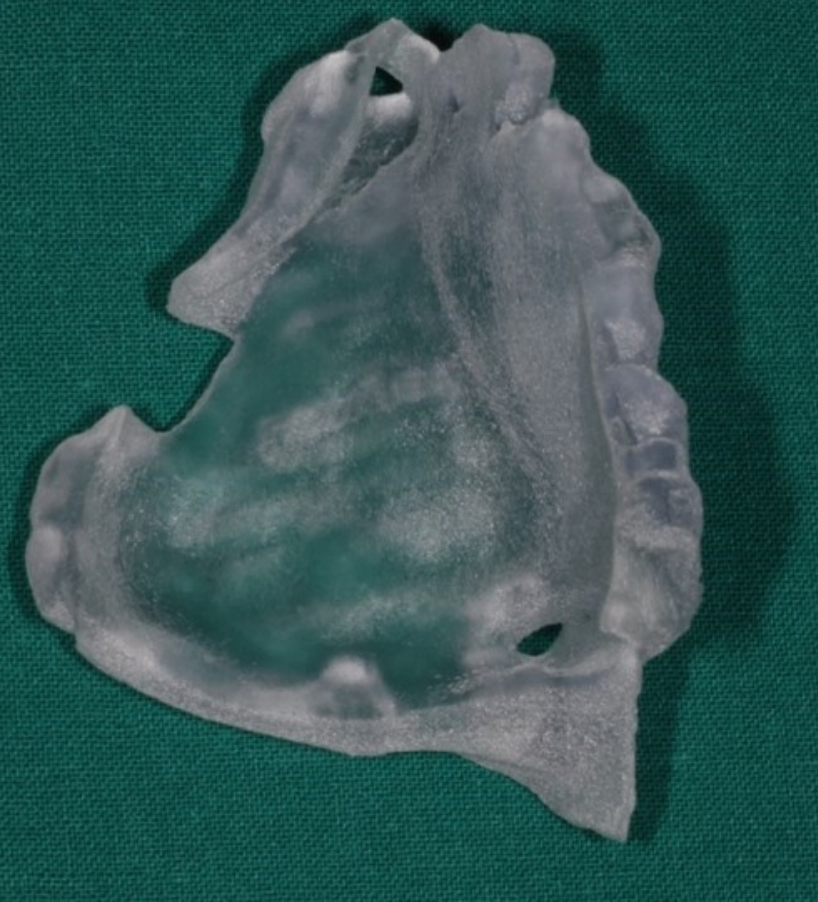




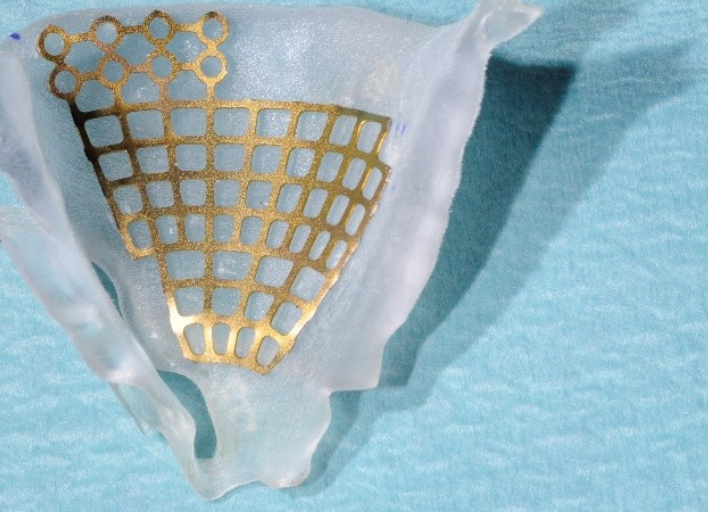





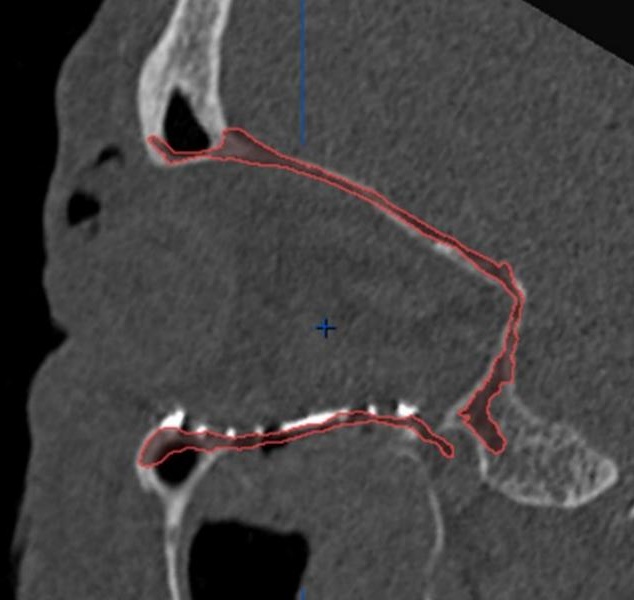





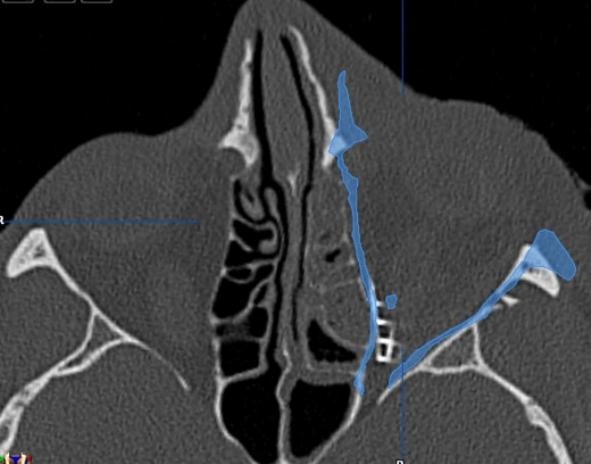




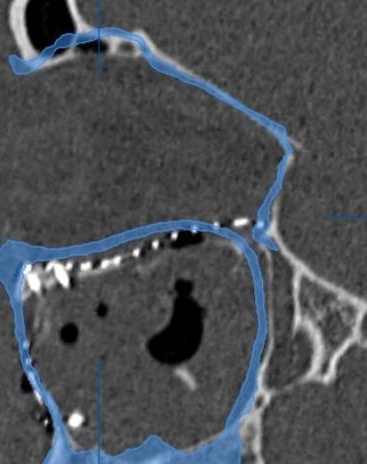




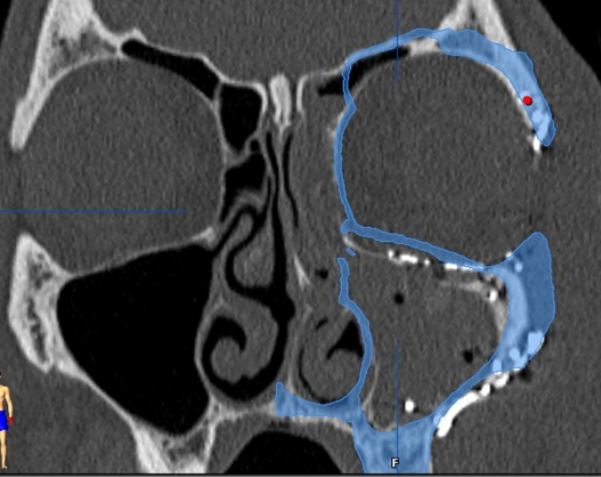




\section{Intraoperative Intension for Revision}

40
35
30
25
20
15
10
5
0

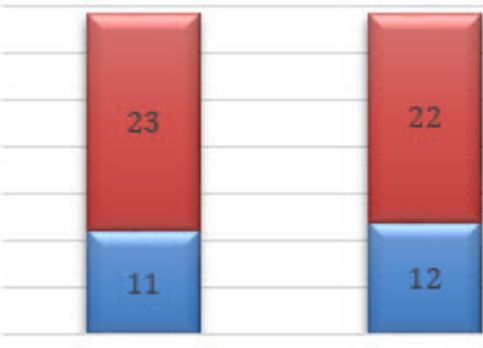

Consultant 1
Consultant 2

a Revision $\mathbf{a}$ No Revision

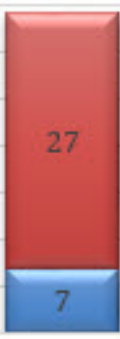

Resident1

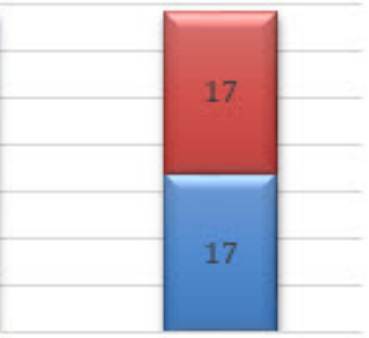

Resident2 


\section{Inpatient Intension for Revision}

40

35

30

25

20

15

10

5

0

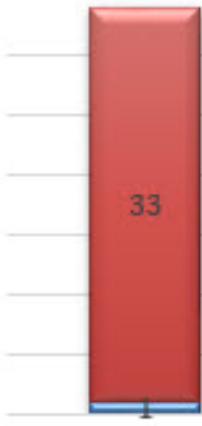

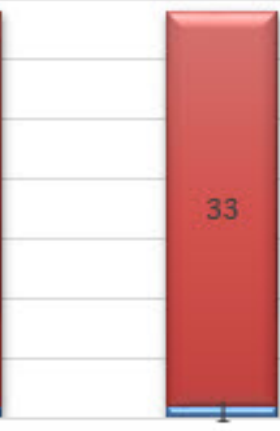

Consultant2

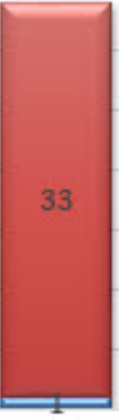

Resident1

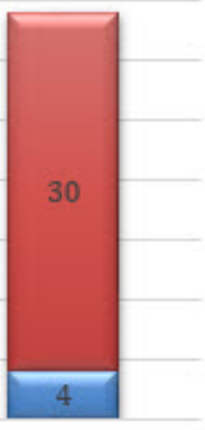

Resident2

QRevision $\mathbf{\Delta}$ No Revision 


\section{Outpatient Intension for Revision}

40

35

30

25

20

15

10

5

0

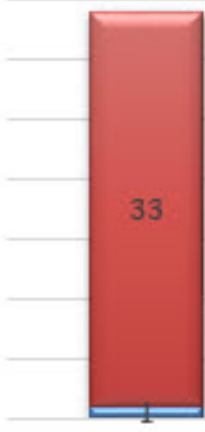

Consultant1

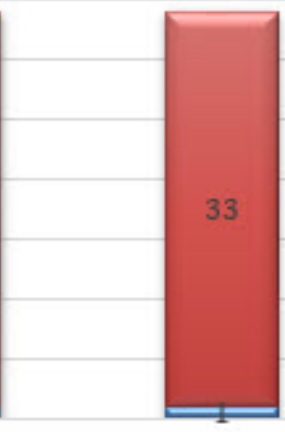

Consultant2

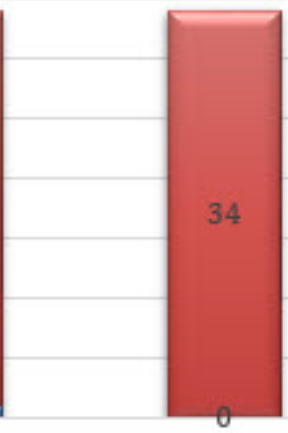

Resident1

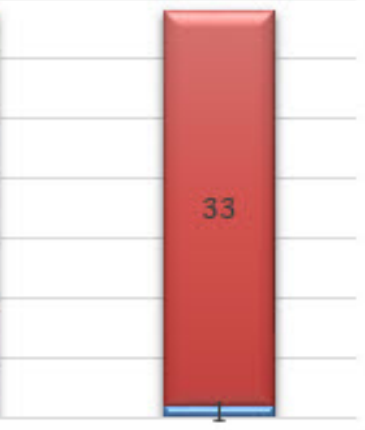

Resident2

घhevision $\square$ No Revision 


\section{Reconstruction Quality}

40

35

30

25

20

15

10

5

0

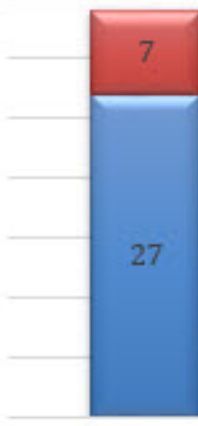

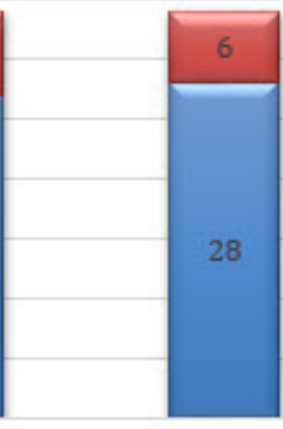

Consultant 2

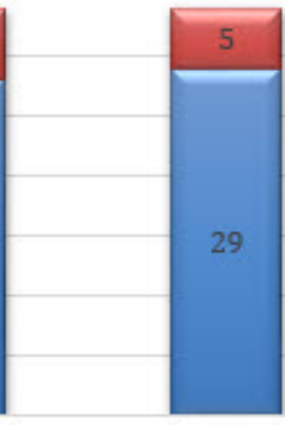

Resident1

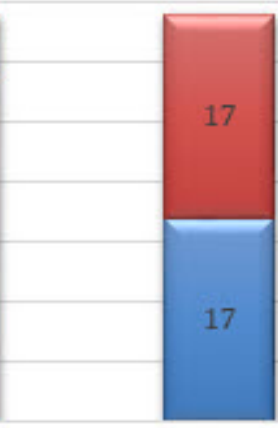

Resident2

-Good $\square$ Poor 